\title{
'Capsule Sparing' Cystoprostadenectomy for Orthotopic Bladder Replacement: Overcoming the Problems of Impotence, Incontinence and Difficult Urethral Anastomosis
}

Ahmed N. Ghanem, MD, FRCSE

Consultant Urologist Surgeon, Mansoura University, No1 President Mubarak Street, Mansoura 35511, Egypt. anmghanem1@gmail.com

*Corresponding Author: Ahmed N. Ghanem, MD, FRCSE, No1 President Mubarak Street, Mansoura 35511, Egypt.

Abstract

Objective: to report a new procedure namely 'capsule sparing' cystoprostadenctomy for orthotopic bladder replacement that overcomes the problems of difficult urethral anastomosis, impotence and incontinence. The question addressed in the present study was whether the objectives of 'nerve-sparing' cystectomy and orthotopic bladder replacement can be preserved, the incidence of incontinence and impotence improved, and surgery made easier and less demanding without compromising the radical excision of cancer.

Patients and methods: The new surgery was implemented on 4 patients with confined bladder cancer as replacementfor'nervesparing'cystprostadenectoy. Thenew techniqueis described in which cystoprostadenctomy for orthotopic bladder replacement while sparing the prostatic capsule.

Results: The objectives of 'nerve-sparing' cystectomy and orthotopic bladder replacement are preserved, the incidence of incontinence and impotence improved, and surgery made easier and less demanding without compromising the radical excision of cancer.

Conclusion: The spared prostatic capsule overcomes the technical difficulty of urethral anastomosis to the neobladder replacement, allowing an easy and robust anastomosis, faster surgery, minimal leakage, no complications and a shorter hospital stay. Preserving potency and continent normal voiding improves the social image and quality of life, both appreciated by the patient.

Keywords: Bladder cancer, Cystoprostadenectomy, Nerve sparing cystectomy, New technique, Capsule sparing

\section{Key PoinTs}

\section{Question}

Can the objectives of 'nerve-sparing' cystectomy and orthotopic bladder replacement be preserved, the incidence of incontinence and impotence improved, and surgery made easier and less demanding without compromising the radical excision of cancer?

\section{Findings}

The point of technique reported here achieves the objectives of 'nerve-sparing' cystectomy and orthotopic bladder replacement while fulfilling the goals mentioned in the question above.

\section{Meaning}

The spared prostatic capsule overcomes the technical difficulty of urethral anastomosis to the neobladder replacement, allowing an easy and robust anastomosis, faster surgery, minimal leakage, no complications and a shorter hospital stay.

\section{INTRODUCTION}

The standard surgical therapy for organ-confined 
'Capsule Sparing' Cystoprostadenectomy for Orthotopic Bladder Replacement: Overcoming the Problems of Impotence, Incontinence and Difficult Urethral Anastomosis

invasive bladder carcinoma is radical cystoprostatectomy ${ }^{1-6}$, which necessitates an internal or external urinary diversion. The ileal conduit has been the 'gold standard' diversion during the last half century ${ }^{7,8}$ despite the availability of arguably better alternatives ${ }^{1,9}$, e.g. orthotopic bladder substitutes, where the urethra can be preserved $^{1-6}$ or internal ureterosigmoidostomy. Most of the problems met in ureterosigmoidostomy occur when the ureters are implanted directly into a peristaltic sphincter-controlled intact bowel tube. This caused the long-term complications of reflux and infection-induced renal damage, metabolic disturbance $^{2}$ and malignant transformation of bowel, mainly of adenocarcinoma ${ }^{10}$ ] but also of other rare tumours ${ }^{11}$. However, not all such problems are relevant to the patient with cancer when using the new methods of diversion of isolated detubularized bowel segments.

Ureteric reflux is managed by detubularizing the bowel segment into pouch and creating an antireflux mechanism either by a nipple valve or one of many tunnelling methods. The submucosal tunnel is commonly used with colon bladders ${ }^{1,3,12}$ and the serous-lined extramural tunnel is the current 'state of the art' used with the ileal bladder ${ }^{4}$. Currently there is almost no reflux and it is less of a problem than anastomotic stricture, affecting $7 \%$ of cases $^{3}$. The issue of the need for an antireflux mechanism was discussed by Studer et $a l^{13}$.

For restoring normal voiding, various bowel segments have been used for constructing an orthotopic bladder pouch substitute either from ileum ${ }^{4}$, caecum and ileum (Mainz) ${ }^{1,3}$, or sigmoid $^{12}$. Genito-reproductive functions were initially ignored, being less important, particularly in theageing Western patientwith cancer ${ }^{1,3}$. However, a younger group of selected patients with bladder cancer ${ }^{5,6,14}$ and the increased tumour-specific 5 -year survival rate of $>75 \%{ }^{1-3}$ highlighted the need to preserve potency in almost all patients with bladder cancer $^{1-6}$ and of reproductive function in selected patients $^{6,14}$. The Walsh 'nerve-sparing' technique ${ }^{15,16}$ for preserving potency was introduced over 17 years ago in radical prostatectomy ${ }^{2}$ and the same technique is used in total cystectomy ${ }^{1}$. At present, impotence affects at least a third of patients after 'nerve-sparing' procedures, even in the most experienced hands ${ }^{2}$, with values of $45 \%{ }^{1}$ and $49 \%{ }^{6}$ reported. The high incidence of impotence was explained by unnoticed damage to the pudendal artery ${ }^{1}$, but another factor responsible was affirmed by the reported anatomical relationship of Denonvillier's fascia with the neurovascular plexus that is not lateralized, scattered and mingled with posterolateral aspects of the fascia, often excised during 'nerve-sparing' cystectomy ${ }^{17}$.

The question addressed in the present study was whether the objectives of 'nerve-sparing' cystectomy and orthotopic bladder replacement can be preserved, theincidence ofincontinenceand impotence improved, and surgery made easier and less demanding without compromising the radical excision of cancer. The feasibility of the new method was encouraged as 'nerve-sparing' modifications do not compromise cancer control $11^{4,18}$, the prostate margin is constantly tumour-free ${ }^{2}$ and total urethrectomy is rarely required in men $^{1}$. Thus this report describes experience with the technique of radical retropubic extraperitoneal cystoprostadenectomy for orthotopicbladder substitution with 'prostate capsule-sparing' that preserves the neurovascular plexus, assuring better potency and continent normal voiding, and making the neo-vesicocapsular anastomosis easier, without compromising radical cancer excision.

\section{Patients And Methods}

The pre-surgical assessment included a chest X-ray, CT, cystoscopy and biopsy for staging the bladder tumour and assuring a tumour-free urethra. Through a lower midline incision and retropubic posterolateral extraperitoneal bladder exposure, dissection was limited to mobilising both ureters, ligating superior vesical vessels and pelvic lymph node excision ${ }^{9}$ (Fig. 1). The postero-lateral surface of the prostate with surrounding Denonvillier's fascia, neurovascular plexus and membranous urethra were left intact, which is the key point in the new technique. Anterior prostatic veins were divided between ligatures and the prostate capsule opened transversely $\approx 0.5 \mathrm{~cm}$ below the bladder neck, as in retropubic prostatectomy. The index finger was used for enucleating the whole prostate adenoma, separating it all around from the 
'Capsule Sparing' Cystoprostadenectomy for Orthotopic Bladder Replacement: Overcoming the Problems of Impotence, Incontinence and Difficult Urethral Anastomosis

capsule, without opening the urethra or disturbing its attachment to the bladder. The urethra was cut transversely below the verumontanum using curved scissors from within the capsule cavity. The intact prostate adenoma with closed urethra was delivered outside the prostatic capsule cavity and kept attached to the intact bladder-containing tumour. A circumferential incision completed the anterior transverse incision of the prostatic capsule. The inferior vesical vessels and seminal vesicles were cut across between ligatures. The cystoprostadenectomy specimen with the full length of the prostatic urethra was removed en bloc, including the peritoneal cover and seminal vesicles, leaving the prostate capsule cup (Fig 2a). A suitable bowel segment on a pedicled blood supply was split longitudinally and constructed into a bladder pouch. The technique for the $\mathrm{W}$-ileal bladder with serous-lined extramural tunnel ${ }^{4}$ and sigmoid neobladder ${ }^{12}$ were used in two patients each (Fig. $2 b)$. The ureters were implanted into the neobladder pouch and posterior suture line(s) closed. The neobladder neck was sutured to the posterior margin of the prostatic capsule. After placing a urethral Foley catheter and exteriorizing the ureteric catheters through the anterior wall of the neobladder and abdomen, the anterior suture line of the capsule and neobladder was completed. The pelvis was drained and the wound closed in layers.

The excised specimen was sent for pathological tumour staging, and examination of the cut prostate margin and urethra. Ureteric reflux, anastomotic leakage and incontinence were assessed by cystography before removing the catheters 2 weeks after surgery. Impotence and incontinence were assessed by direct enquiry before leaving hospital and during the follow-up.

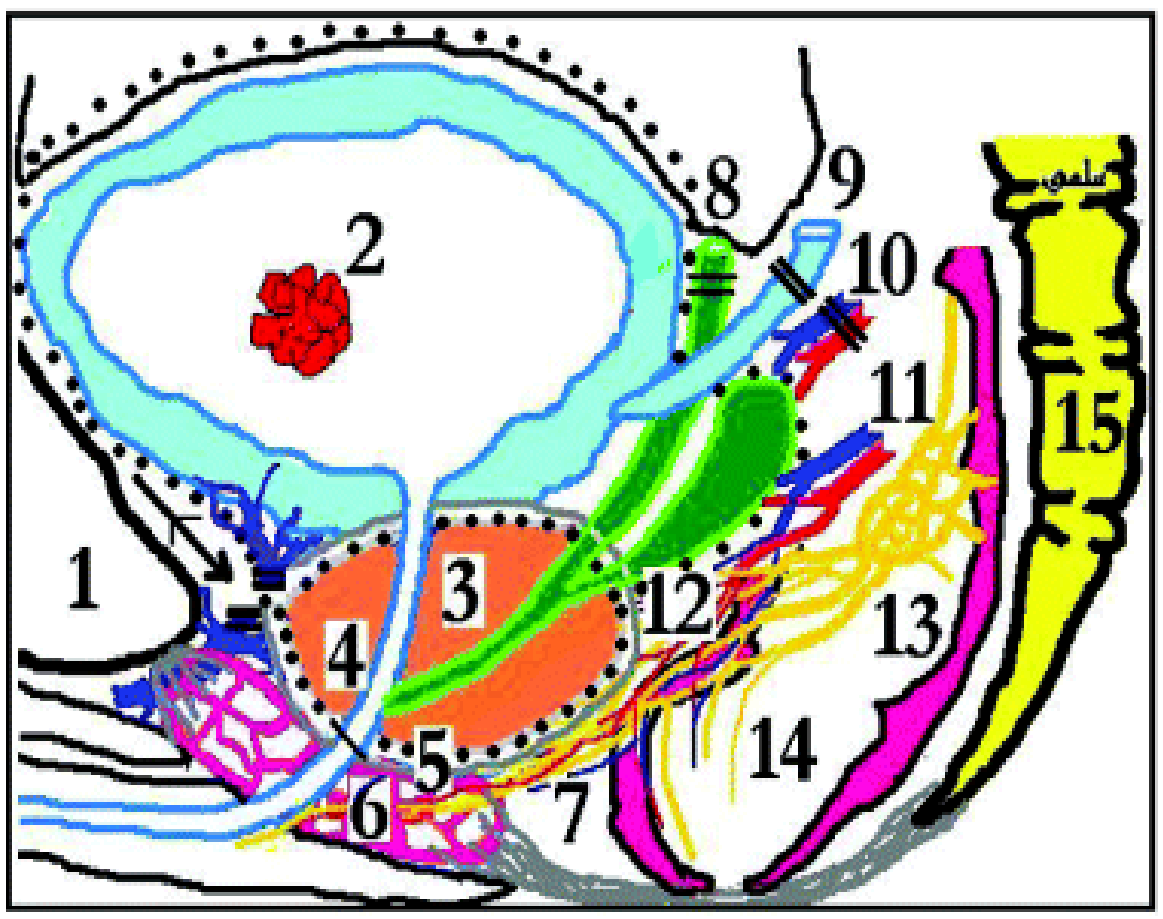

Figure 1. A diagramatic representation of the technique's dissection plane (dotted line). Starting at retro-pubic (1) space, visico-prost-adenectomy including bladder containing tumour (2), prostate gland (3) and urethra (4) are excised in mass. The gland is enucleated and urethral divided within the spared capsule (5) protecting the external sphincter (6) and neurovascular bundle (7). Extra-peritoneal division ligature of vas (8), ureter (9), superior vesical vessels (10) and anterior prostatic veins are done at the start. Branches of inferior vesical vessels (11) and seminal vesicles (12) are divided at the line of capsule division. Excision of peritoneal bladder cover opens peritoneal cavity for constructing bowel bladder replacement. Pelvic ganglion (13) and rectum (14) lie within the concavity of sacrum (15). 
'Capsule Sparing' Cystoprostadenectomy for Orthotopic Bladder Replacement: Overcoming the Problems of Impotence, Incontinence and Difficult Urethral Anastomosis

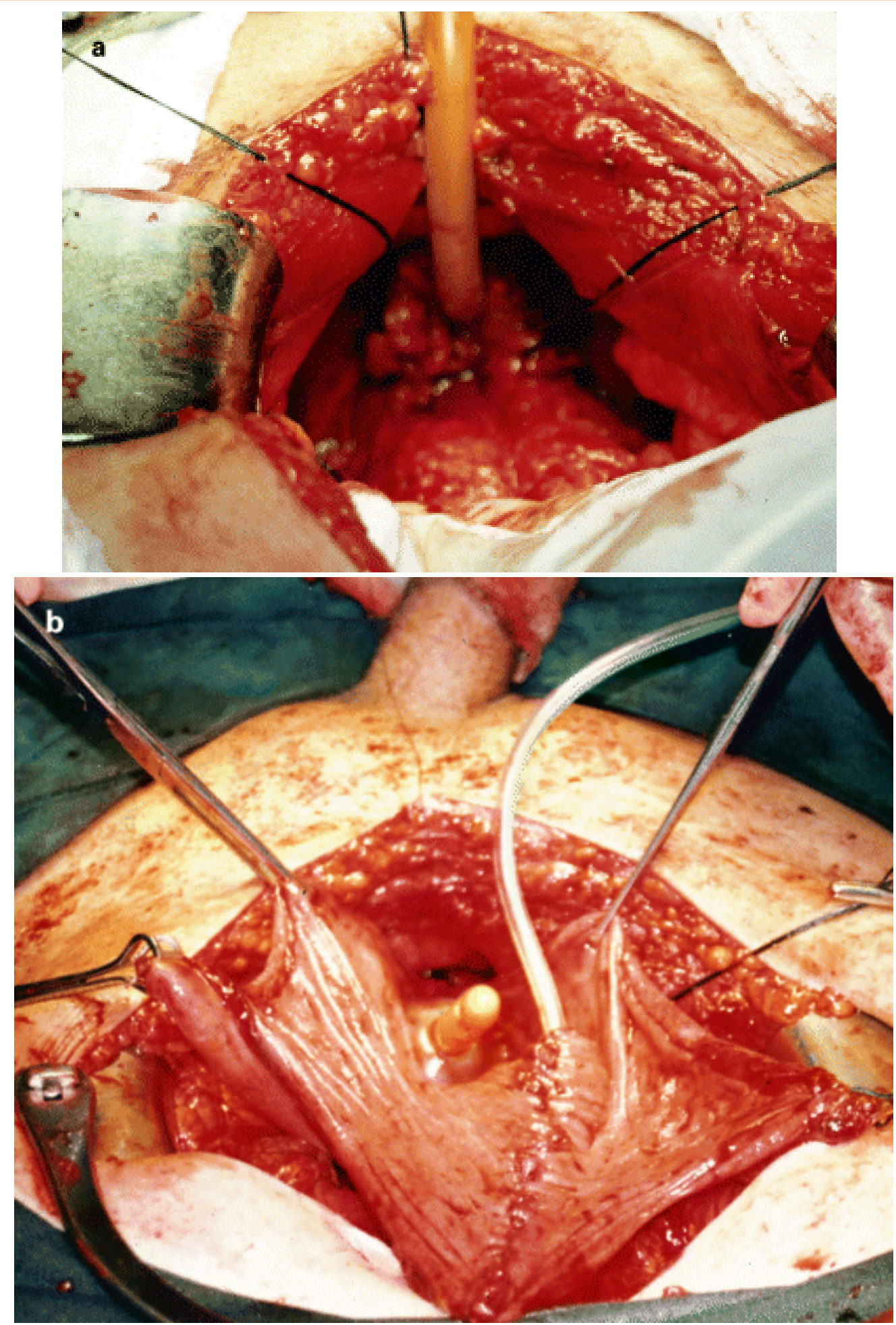

Figure 2. $a$, Spared prostatic capsule cup with the Foley catheter in the cavity and black silk sutures marking its margin, allowing easy access and a robust neobladder-to-capsule anastomosis. b, Neo-orthotopic sigmoid bladder shaped into inverted $U$ pouch. The catheterized single ureter is implanted, posterior suture completed and the neo-bladder neck sutured to the posterior margin of prostate capsule. The Foley catheter balloon occupies the capsule cavity. 
'Capsule Sparing' Cystoprostadenectomy for Orthotopic Bladder Replacement: Overcoming the Problems of Impotence, Incontinence and Difficult Urethral Anastomosis

\section{RESULTS}

The cystoprostadenectomy was as radical as standard cystectomy, as all removed specimens had a tumour-free cut margin and full-length prostatic urethra. The spared prostatic capsule cup allowed easy access and robust anastomosis to the neobladder substitute. The procedure was undertaken in four patients (mean age 47 years, SD 5.2) between 1994 and 2000, with no mortality or morbidity. The mean (SD) duration of surgery was $4.7(0.9) \mathrm{h}$. Three patients had TCC and one squamous cell carcinoma (SCC). The tumour was bladder-confined (pT2N0M0) in all except the patient with SCC, whose tumour invaded the bladder adventitia (T3). Three patients survived at a mean (SD, range) follow-up of 3.6 (1.8, 1.2-6) years. The patient with SCC had local pelvic recurrence after 6 months and died within 2 years.

Cystography for reflux, anastomotic leakage and incontinence was negative and showed a neobladder capacity of $>500 \mathrm{~mL}$. After removing the Foley and ureteric catheters, the patients' potency was assessed by direct enquiry and response to tactile stimulation, and incontinence further assessed. All patients were dry day and night and all but one uraemic patient reported erection before leaving hospital within 3 weeks of surgery, and successful intercourse at home. The uraemic patient did not, as he progressed into end-stage renal failure requiring dialysis 3 months after surgery. This patient had TCC involving his renal collecting system, ureter and bladder and that destroyed his right kidney; he also had hydronephrosis of the left kidney from a chronic bilharzial lower ureteric stricture, visible on IVU, but CT exclu ded lymph node enlargement and liver metastasis. He underwent total nephroureterectomy and cystoprostadenectomy at the same setting (Figure 3). After excising the stricture, the left small hydroureter was directly implanted into the neobladder. His residual left renal function deteriorated into end-stage renal failure. One patient was newly married and had his seminal vesicles, vasa deferentia and potency preserved for future fertility, using semen retrieval from urine for artificial insemination.

\section{Discussion}

This reported modification transfers the plane of dissection into the prostate capsule, assuring preservation of the neurovascular plexus, potency and continence. The key to the technique is avoiding handling the neurovascular plexus ${ }^{17}$ and the sphincter-controlled membranous urethra, to fully preserve potency and continence, respectively. The spared prostatic capsule also allows easy and robust anastomosis to the neobladder, reducing the duration of surgery and complications without compromising the radical excision of cancer.

Cystoprostadenectomy has the same indications and limitations as 'nerve-sparing' cystectomy ${ }^{1,2,16}$.The current technique of extracapsular 'nerve-sparing' reported previously ${ }^{2}$ and applied to total cystectomy ${ }^{1}$ has a stable incidence for both incontinence ${ }^{1,4}$ and impotence ${ }^{1,2,6,18-20}$, i.e. a third and a half respectively, irrespective of the meticulousness of technique and experience. From the anatomy ${ }^{17}$ further progress is only possible by leaving undisturbed the area of Denonvillier's fascia, neurovascular plexus and sphincter-controlled membranous urethra, giving the key principles of 'prostate capsule sparing' to assure the preservation of potency and continence while allowing easy access and a robust anastomosis. The procedure is easier and less demanding than previous methods

Potency was preserved in three patients and impaired in one, probably by uraemia rather than the procedure. The patient had TCC involving his right kidney, ureter and bladder (Fig. 3) and left hydronephrosis that progressed to renal failure. Although there were only four patients in the presentstudy, this is acceptable ${ }^{6}$ and most previous modifications were reported on 4-10 patients $^{6,12,18,19}$. A similar technique based on TUR of prostatic adenoma followed by open 'capsule-sparing' cystectomy was reported in eight patients, with all having preserved potency and continence ${ }^{12}$. However, such a TUR technique may increase the complexity and time required for surgery, as well as carrying a possible risk of cancer cell implantation.

The present method overcomes the technical difficulty of urethral anastomosis to the neobladder replacement, allowing an easy and robust anastomosis, faster surgery, minimal leakage, no complications and a shorter hospital stay. The spared prostatic capsule cup augments the neobladder pouch capacity and protects the outlying neurovascular plexus, assuring a minimal incidence of impotence. Avoiding direct 
'Capsule Sparing' Cystoprostadenectomy for Orthotopic Bladder Replacement: Overcoming the Problems of Impotence, Incontinence and Difficult Urethral Anastomosis

handling of the membranous urethra protects the controlling action of the surrounding sphincter muscle, preventing incontinence. Preserving potency and continent normal voiding improves the social image and quality of life, both appreciated by the patient (Fig. 4). The advantages of the present technique to patients, evident soon after surgery and affirmed on follow-up, may increase the popularity of an already better procedure.
The method removed the bladder with a closed bladder neck, a full length prostatic urethra and adenoma, allowing a larger safety margin than is possible during the resection of cancer bladder from the adjacent pelvic structures. The present technique does not compromise radical cancer excision, particularly as long-term results show that the prostatic margin is always tumour-free ${ }^{2}$ and that the urethra is rarely involved in men ${ }^{1}$.

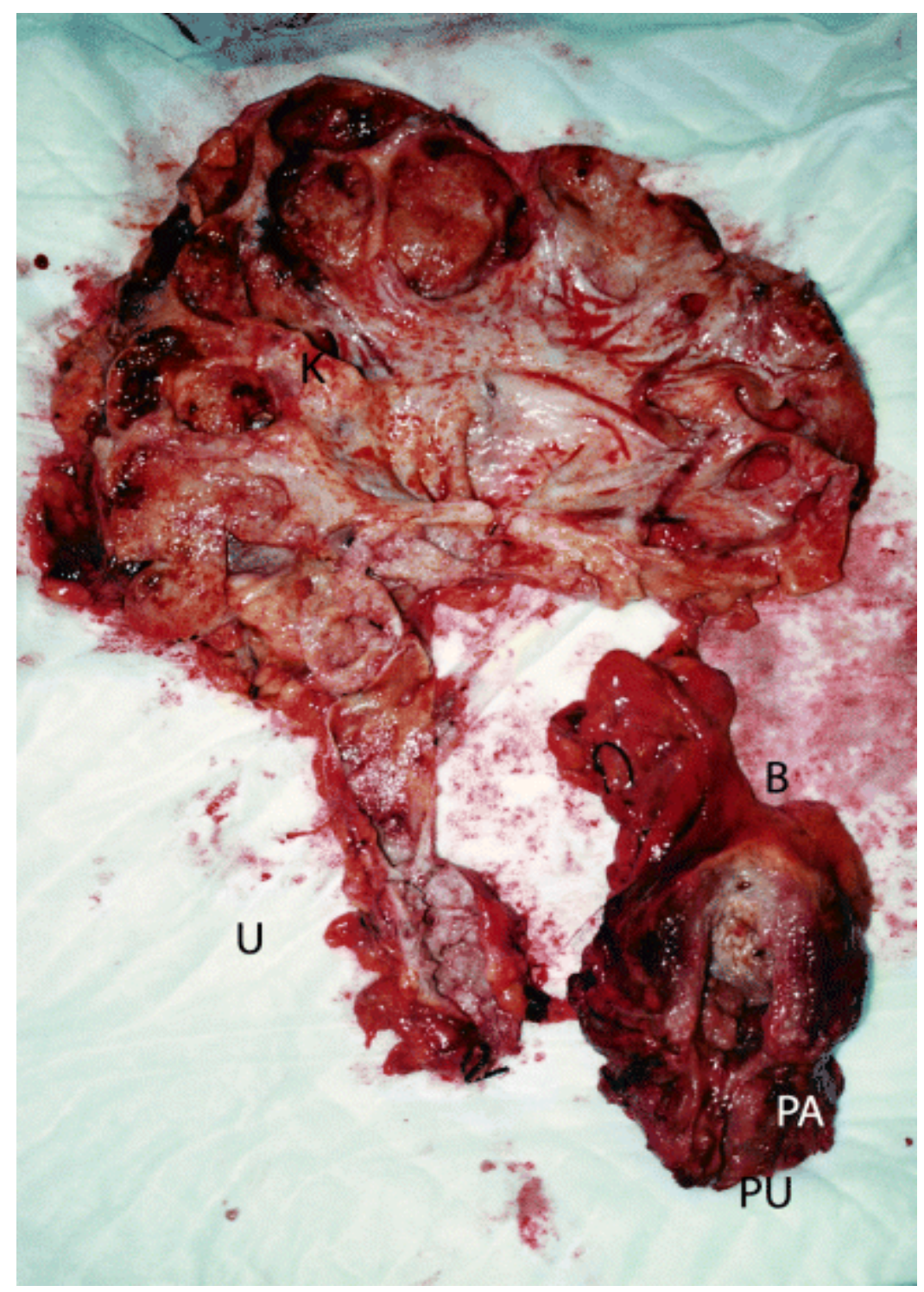

Figure 3. Nephroureterectomy and cystoprostadenectomy specimens excised at the same sitting, showing the tumour involving the kidney $(K)$, ureter $(U)$ and bladder $(B)$. The cut edge of prostate adenoma (PA) and urethra $(P U)$ extending below the verumontanum was tumour-free. Despite the extensive epithelial spread of transitional cell tumour, the stage was pT2NOMO. Cystoprostadenectomy with 'prostate capsule sparing' is as radical as total cystectomy but assures preservation of the neurovascular plexus and sphincter-controlled membranous urethra, preventing impotence and incontinence. 
'Capsule Sparing' Cystoprostadenectomy for Orthotopic Bladder Replacement: Overcoming the Problems of Impotence, Incontinence and Difficult Urethral Anastomosis

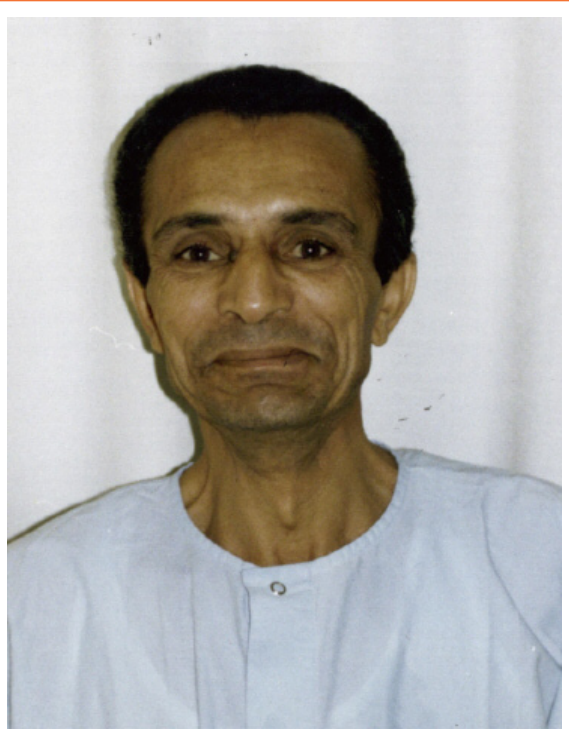

Figure 4. Shows a patient at the post-operative period, on whom the reported procedure (Fig. 2) was performed, expressing his appreciation for the result.

He gave consent to photography and publication.

\section{REFERENCES}

[1] Venn, SN, Popert, RM, Mundy, AR. 'Nerve sparing' cystectomy and substitution cystoplasty in patients of either sex. Limitations and techniques. Br J Urol 1998; 82:361- 5

[2] Brendler, CB, Steinberg, GD, Marshall, FF, Mostwin, JL, Walsh, PC. Local recurrence and survival following nerve-sparing radical cystoprostatectomy. J Urol 1990; 144:1137- 40

[3] Leisner, I, Stein, R, Hohenfeller, R et al. Radical cystoprostatectomy combined with Mains pouch bladder substitution to the urethra: long-term results. BJU Int 1999; 83:964- 70

[4] Aboul-Enein, H, Ghoneim, MA. A novel uretero-ileal re-implantation technique: the serous lined extramural tunnel. A preliminary report. J Urol 1994; 151: 1193- 7

[5] Koraitim, M, Khalil, R. Preservation of urosexual functions after radical cystectomy. Urology 1992; 39: 117- 21

[6] Spitz, A, Stein, JP, Lieskovsky, G, Skinner, DG. Orthotopic urinary diversion with preservation of erectile and ejaculatory function in men requiring radical cystectomy for nonurothelial malignancy: a new technique. $J$ Urol 1999; 161: 1761- 4

[7] Whittlestone, TH, Persad, R. Radical cystectomy and bladder substitution. Hosp Med 2000; 61: 336- 40

[8] Moffat, L. Radical cystectomy and ileal conduit for invasive bladder tumour. $J R$ Coll Surg Edinb 1999; 44: 379- 85

[9] Ghanem, AN. Radical cystectomy and ileal conduit for invasive bladder tumour. J R Coll Surg Edinb 2000; 45: 204- 5

[10] Stewart, M. Urinary Diversion and Cancer. Thesis. University of London, 1984

[11] Ghanem, AN, Perry, KC. Malignant lymphoma as a complication of uretero-sigmoidostomy. $\mathrm{Br} J$ Surg 1985; 72: 559- 60

[12] Grasset, D, Delbos, O, Muir, GH, Robert, M, Guiter, J. Orthotopic bladder substitution by detubularized sigmoid using a new method of neovesico-urethral anastomosis. $\mathrm{Br} J$ Urol 1998; 81: 623- 7

[13] Studer, UE, Danuser, H, Hochreiter, W, Springer, JP, Turner, WH, Zing, EH.Summary of 10 years' experience with an ileal low-pressure bladder substitute combined with an afferent tubular isoperistaltic segment. World $J$ Urol 1996; 14: 29- 34

[14] Colombo, R, Bertini, R, Salonia, A et al. Nerve and seminal sparing radical cystectomy with orthotopic urinary diversion for select patients with superficial bladder cancer: an innovative surgical approach. J Urol 2001; 165: 65- 6

[15] Catalona, WJ. Nerve-sparing radical retropubic prostatectomy. Urol Clin North Am1985; 12: 187- 99

[16] Burgers, JK, Brendler, CB. Anatomic radical cystoprostatectomy. Urol Clin North Am 1991; 18: 659- 76

[17] Kourambas, J, Angus, P, Hosking, P, Chou, ST. A histological study of Denonvillier's fascia and its relation to the neurovascular bundle. $\mathrm{Br} \mathrm{J}$ Urol 1998; 82: 408- 10 
'Capsule Sparing' Cystoprostadenectomy for Orthotopic Bladder Replacement: Overcoming the Problems of Impotence, Incontinence and Difficult Urethral Anastomosis

[18] Chiang, $\mathrm{PH}, \mathrm{Wu}, \mathrm{WJ}$, Chiang, CP.Nerve-sparing radical cystoprostatectomy: 3-year experience. Kaohsiung J Med Sci 1997; 13: 169- 7

[19] Arai, Y, Yoshida, O. En bloc radical cystoprostatourethrectomy with preservation of sexual function. Int J Urol 1995; 2: 249- 51
[20] Miyao, N, Adachi, H, Sato, Y et al. Recovery of sexual function after nerve-sparing radical prostatectomy or cystectomy. Int $J$ Urol 2001; 8: 158- 64

Citation: Ahmed N. Ghanem. 'Capsule Sparing' Cystoprostadenectomy for Orthotopic Bladder Replacement: Overcoming the Problems of Impotence, Incontinence and Difficult Urethral Anastomosis. Archives of Urology. 2019; 2(2): 24-31.

Copyright: (C) 2019 Ahmed N. Ghanem. This is an open access article distributed under the Creative Commons Attribution License, which permits unrestricted use, distribution, and reproduction in any medium, provided the original work is properly cited. 